\title{
New Inner Product Quasilinear Spaces on Interval Numbers
}

\author{
Hacer Bozkurt ${ }^{1}$ and Yilmaz Yilmaz ${ }^{2}$ \\ ${ }^{1}$ Department of Mathematics, Batman University, 72100 Batman, Turkey \\ ${ }^{2}$ Department of Mathematics, İnönü University, 44280 Malatya, Turkey \\ Correspondence should be addressed to Hacer Bozkurt; hacer.bozkurt@batman.edu.tr
}

Received 22 January 2016; Accepted 23 March 2016

Academic Editor: Adrian Petrusel

Copyright (C) 2016 H. Bozkurt and Y. Yilmaz. This is an open access article distributed under the Creative Commons Attribution License, which permits unrestricted use, distribution, and reproduction in any medium, provided the original work is properly cited.

Primarily we examine the new example of quasilinear spaces, namely, " $I \mathbb{R}^{n}$ interval space." We obtain some new theorems and results related to this new quasilinear space. After giving some new notions of quasilinear dependence-independence and basis on quasilinear functional analysis, we obtain some results on $I \mathbb{R}^{n}$ interval space related to these concepts. Secondly, we present $I s, I c_{0}, I l_{\infty}$, and $I l_{2}$ quasilinear spaces and we research some algebraic properties of these spaces. We obtain some new results and provide an important contribution to the improvement of quasilinear functional analysis.

\section{Introduction}

In 1986, Aseev generalized the notion linear spaces by introducing firmly quasilinear spaces. He used the partial order relation when he defined quasilinear spaces and then he can give consistent counterpart of results in linear spaces. For more details, the reader can refer to [1]. This work has motivated a lot of authors to introduce new results on setvalued analysis $[2,3]$.

One of the most useful examples of a quasilinear space is the set $\Omega_{C}(E)$ of all convex compact subsets of a normed space $E$. The investigation of this class involves a convex interval analysis. Intervals are excellent tools for handling global optimization problems and for supplementing standard techniques. This is because an interval is an infinite set and is thus a carrier of an infinite amount of information which means global information. Further, the theory of set differential equations also needs the analysis of $\Omega_{C}(E)$ [3].

Inspired and motivated by research going on in this area, we introduce an inner product quasilinear space which is defined in [4]. Generally, in [4], we give some examples of quasi-inner product properties on $\Omega_{C}(E)$ of all convex compact subsets of a normed space $E$. In our present paper we examine a new type of a quasilinear space, namely, $I \mathbb{R}^{n}$. We find some important results related to the geometric structure of the $I \mathbb{R}^{n}$ quasilinear space and we examine some algebraic properties of the $I \mathbb{R}^{n}$ interval space. Furthermore, we explore the concepts of $I s, I c_{0}, I l_{\infty}$, and $I l_{2}$ interval sequence spaces as new examples of quasilinear spaces. Moreover, we obtain some theorems and results related to these new spaces which provide us with improving the elements of the quasilinear functional analysis.

\section{Quasilinear Spaces and Hilbert Quasilinear Spaces}

Let us start this section by introducing the definition of a quasilinear space and some of its basic properties given by Aseev [1].

Definition 1. A set $X$ is called a quasilinear space if a partial order relation " $\leq$," an algebraic sum operation, and an operation of multiplication by real numbers are defined in it in such way that the following conditions hold for any elements $x, y, z, v \in X$ and any real numbers $\alpha, \beta \in E^{1}$ :
(1) $x \leq x$
(2) $x \leq z$ if $x \leq y$ and $y \leq z$,
(3) $x=y$ if $x \leq y$ and $y \leq x$,
(4) $x+y=y+x$,
(5) $x+(y+z)=(x+y)+z$ 
(6) there exists an element $\theta \in X$ such that $x+\theta=x$,

(7) $\alpha \cdot(\beta \cdot x)=(\alpha \cdot \beta) \cdot x$,

(8) $\alpha \cdot(x+y)=\alpha \cdot x+\alpha \cdot y$,

(9) $1 \cdot x=x$,

(10) $0 \cdot x=\theta$,

(11) $(\alpha+\beta) \cdot x \leq \alpha \cdot x+\beta \cdot x$,

(12) $x+z \leq y+v$ if $x \leq y$ and $z \leq v$,

(13) $\alpha \cdot x \leq \alpha \cdot y$ if $x \leq y$.

A linear space is a quasilinear space with the partial order relation " =." The most popular example which is not a linear space is the set of all closed intervals of real numbers with the inclusion relation " $\subseteq$," algebraic sum operation

$$
A+B=\{a+b: a \in A, b \in B\},
$$

and the real-scalar multiplication

$$
\lambda A=\{\lambda a: a \in A\} .
$$

We denote this set by $\Omega_{C}(\mathbb{R})$. Another one is $\Omega(\mathbb{R})$, the set of all compact subsets of real numbers. By a slight modification of algebraic sum operation (with closure) such as

$$
A+B=\overline{\{a+b: a \in A, b \in B\}}
$$

and by the same real-scalar multiplication defined above and by the inclusion relation we get the nonlinear QLS, $\Omega_{C}(E)$ and $\Omega(E)$, the space of all nonempty closed bounded and convex closed bounded subsets of some normed linear space $E$, respectively.

Lemma 2. Suppose that any element $x$ in a QLS $X$ has an inverse element $x^{\prime} \in X$. Then the partial order in $X$ is determined by equality, the distributivity conditions hold, and, consequently, $X$ is a linear space [1].

Suppose that $X$ is a QLS and $Y \subseteq X$. Then $Y$ is called a subspace of $X$ whenever $Y$ is a QLS with the same partial order and the restriction to $Y$ of the operations on $X$. One can easily prove the following theorem using the condition of being a QLS. It is quite similar to its linear space analogue.

Theorem 3. $Y$ is a subspace of a QLS $X$ if and only if, for every $x, y \in Y$ and $\alpha, \beta \in R, \alpha x+\beta y \in Y$ [5].

Let $X$ be a QLS. An $x \in X$ is said to be symmetric if $-x=x$, where $-x=(-1) x$, and $X_{d}$ denotes the set of all such elements. $\theta$ denotes the zero's additive unit of $X$ and it is minimal, that is, $x=\theta$ if $x \leq \theta$. An element $x^{\prime}$ is called inverse of $x$ if $x+x^{\prime}=\theta$. The inverse is unique whenever it exists and $x^{\prime}=-x$ in this case. Sometimes $x^{\prime}$ may not exist but $-x$ is always meaningful in QLSs. An element $x$ possessing an inverse is called regular; otherwise it is called singular. For a singular element $x$ we should note that $x-x \neq 0$. Now, $X_{r}$ and $X_{s}$ stand for the sets of all regular and singular elements in $X$, respectively. Further, $X_{r}, X_{d}$, and $X_{s} \cup\{0\}$ are subspaces of $X$ and they are called regular, symmetric, and singular subspaces of $X$, respectively [5]. It is easy from the definitions that $X_{d} \subset X_{s}$ and $X=X_{r} \cup X_{s}$.

In a linear QLS that is in a linear space, there is no singular element. Further, in a QLS $X$, it is obvious that any element $x$ is regular if and only if $x-x=0$.

Proposition 4. In a $Q L S X$, if $x \in X$ and $y \in X_{s}$ then $x+y \in$ $X_{s}$.

Proposition 5. In a quasilinear space $X$ every regular element is minimal [5].

Definition 6. Let $X$ be a QLS and $y \in X$. The set of all regular elements preceding $y$ is called floor of $y$, and $F_{y}$ denotes the set of all such elements. Therefore,

$$
F_{y}=\left\{z \in X_{r}: z \leq y\right\}
$$

The floor of any subset $M$ of $X$ is the union of floors of all elements in $M$ and is denoted by $F_{M}[6]$.

Definition 7. Let $X$ be a QLS, $M \subseteq X$ and $x, y \in M . M$ is called a proper set if the following two conditions hold:

(i) $F_{z} \neq \emptyset$ for all $z \in M$,

(ii) $F_{x} \neq F_{y}$ for each pair of points $x, y$ with $x \neq y$.

Otherwise $M$ is called an improper set. Particularly if $X$ is a proper set, then it is called a proper quasilinear space (briefly, proper qls) [6].

Definition 8. Let $X$ be a quasilinear space. $X$ is called a solidfloored quasilinear space whenever

$$
y=\sup \left\{x \in X_{r}: x \leq y\right\}
$$

for each $y \in X$. Otherwise, $X$ is called a non-solid-floored quasilinear space [6].

Definition 9. Let $X$ be a QLS. Consolidation of floor of $X$ is the smallest solid-floored QLS $\widehat{X}$ containing $X$, that is, if there exists another solid-floored QLS $Y$ containing $X$ then $\widehat{X} \subseteq Y$.

Clearly, $\widehat{X}=X$ for some solid-floored QLS $X$. Further, $\widehat{\Omega_{C}\left(\mathbb{R}^{n}\right)_{s}}=\Omega_{C}\left(\mathbb{R}^{n}\right)$. For a QLS $X$, the set

$$
F_{y}^{\widehat{X}}=\left\{z \in(\widehat{X})_{r}: z \preceq y\right\} .
$$

is the floor of $X$ in $\widehat{X}$.

Definition 10. Let $X$ be a QLS. A real function $\|\cdot\|_{X}: X \rightarrow E^{1}$ is called a norm if the following conditions hold [1]:

(14) $\|x\|_{X}>0$ if $x \neq 0$,

(15) $\|x+y\|_{X} \leq\|x\|_{X}+\|y\|_{X}$,

(16) $\|\alpha x\|_{X}=|\alpha|\|x\|_{X}$,

(17) if $x \leq y$, then $\|x\|_{X} \leq\|y\|_{X}$,

(18) if for any $\varepsilon>0$ there exists an element $x_{\varepsilon} \in X$ such that $x \leq y+x_{\varepsilon}$ and $\left\|x_{\varepsilon}\right\|_{X} \leq \varepsilon$ then $x \leq y$. 
A quasilinear space $X$ with a norm defined on it is called normed QLS. It follows from Lemma 2 that if any $x \in X$ has an inverse element $x^{\prime} \in X$, then the concept of a normed QLS coincides with the concept of a real normed linear space.

Let $X$ be a normed QLS. Hausdorff or norm metric on $X$ is defined by the equality

$$
\begin{aligned}
& h_{X}(x, y) \\
& \quad=\inf \left\{r \geq 0: x \leq y+a_{1}^{r}, y \leq x+a_{2}^{r},\left\|a_{i}^{r}\right\| \leq r\right\} .
\end{aligned}
$$

Since $x \leq y+(x-y)$ and $y \leq x+(y-x)$, the quantity $h_{X}(x, y)$ is well defined for any elements $x, y \in X$, and

$$
h_{X}(x, y) \leq\|x-y\|_{X} .
$$

It is not hard to see that this function $h_{X}(x, y)$ satisfies all of the metric axioms.

Lemma 11. The operations of algebraic sum and multiplication by real numbers are continuous with respect to the Hausdorff metric. The norm is a continuous function with respect to the Hausdorff metric [1].

Example 12. Let $E$ be a Banach space. A norm on $\Omega(E)$ is defined by

$$
\|A\|_{\Omega(E)}=\sup _{a \in A}\|a\|_{E} .
$$

Then $\Omega(E)$ and $\Omega_{C}(E)$ are normed quasilinear spaces. In this case the Hausdorff metric is defined as usual:

$$
\begin{aligned}
& h_{\Omega_{C}(E)}(A, B) \\
& \quad=\inf \left\{r \geq 0: A \subset B+S_{r}(\theta), B \subset A+S_{r}(\theta)\right\},
\end{aligned}
$$

where $S_{r}(\theta)$ denotes a closed ball of radius $r$ about $\theta \in X[1]$.

Let us give an extended definition of inner-product. This definition and some prerequisites are given by Y. Yllmaz. We can see following inner-product as (set-valued) inner product on QLSs.

Definition 13. Let $X$ be a quasilinear space. A mapping $\langle\cdot, \cdot\rangle$ : $X \times X \rightarrow \Omega(\mathbb{R})$ is called an inner product on $X$ if for any $x, y, z \in X$ and $\alpha \in \mathbb{R}$ the following conditions are satisfied:

(19) if $x, y \in X_{r}$ then $\langle x, y\rangle \in \Omega_{C}(\mathbb{R})_{r} \equiv \mathbb{R}$,

(20) $\langle x+y, z\rangle \subseteq\langle x, z\rangle+\langle y, z\rangle$,

(21) $\langle\alpha \cdot x, y\rangle=\alpha \cdot\langle x, y\rangle$,

(22) $\langle x, y\rangle=\langle y, x\rangle$,

(23) $\langle x, x\rangle \geq 0$ for $x \in X_{r}$ and $\langle x, x\rangle=0 \Leftrightarrow x=0$,

(24) $\|\langle x, y\rangle\|_{\Omega(\mathbb{R})}=\sup \left\{\|\langle a, b\rangle\|_{\Omega(\mathbb{R})}: a \in F_{x}^{\widehat{X}}, b \in F_{y}^{\widehat{X}}\right\}$,

(25) if $x \preceq y$ and $u \preceq v$ then $\langle x, u\rangle \subseteq\langle y, v\rangle$,

(26) if for any $\varepsilon>0$ there exists an element $x_{\varepsilon} \in X$ such that $x \preceq y+x_{\varepsilon}$ and $\left\langle x_{\varepsilon}, x_{\varepsilon}\right\rangle \subseteq S_{\varepsilon}(\theta)$ then $x \preceq y$.

A quasilinear space with an inner product is called an inner product quasilinear space, briefly IPQLS.
Example 14. One can see easily $\Omega_{C}(\mathbb{R})$, the space of closed real intervals, is a IPQLS with inner-product defined by

$$
\langle A, B\rangle=\{a \cdot b: a \in A, b \in B\} .
$$

Every IPQLS $X$ is a normed QLS with the norm defined by

$$
\|x\|=\sqrt{\|\langle x, x\rangle\|_{\Omega(\mathbb{R})}}
$$

for every $x \in X[4]$.

Proposition 15. If in an IPQLS $x_{n} \rightarrow x$ and $y_{n} \rightarrow y$, then $\left\langle x_{n}, y_{n}\right\rangle \rightarrow\langle x, y\rangle[4]$.

AN IPQLS is called Hilbert QLS, if it is complete according to the Hausdorff (norm) metric.

Example 16. Let $E$ be an inner product space. Then we know that $\Omega(E)$ is an IPQLS and it is complete with respect to the Hausdorff metric. So, $\Omega(E)$ is a Hilbert QLS [4].

Definition 17 (orthogonality). An element $x$ of an IPQLS $X$ is said to be orthogonal to an element $y \in X$ if

$$
\|\langle x, y\rangle\|_{\Omega(\mathbb{R})}=0 .
$$

We also say that $x$ and $y$ are orthogonal and we write $x \perp y$. Similarly, for subsets $m, n \subseteq X$ we write $x \perp m$ if $x \perp z$ for all $z \in m$ and $m \perp n$ if $a \perp b$ for all $a \in m$ and $b \in n$ [4].

An orthonormal set $M \subset X$ is an orthogonal set in $X$ whose elements have norm 1 ; that is, for all $x, y \in M$

$$
\|\langle x, y\rangle\|_{\Omega(\mathbb{R})}= \begin{cases}0, & x \neq y \\ 1, & x=y .\end{cases}
$$

Definition 18. Let $A$ be a nonempty subset of an inner product quasilinear space $X$. An element $x \in X$ is said to be orthogonal to $A$, denoted by $x \perp A$, if $\|\langle x, y\rangle\|_{\Omega(\mathbb{R})}=0$ for every $y \in A$. The set of all elements of $X$ orthogonal to $A$, denoted by $A^{\perp}$, is called the orthogonal complement of $A$ and is indicated by

$$
A^{\perp}=\left\{x \in X:\|\langle x, y\rangle\|_{\Omega(\mathbb{R})}=0, y \in A\right\} .
$$

Theorem 19. For any subset $A$ of an IPQLS X, $A^{\perp}$ is a closed subspace of $X[4]$.

Definition 20. Let $X$ be a quasilinear space, $\left\{x_{k}\right\}_{k=1}^{n} \subset X$. The element $x \in X$ with

$$
\alpha_{1} x_{1}+\alpha_{2} x_{2}+\cdots+\alpha_{n} x_{n} \leq x
$$

is said to be a quasilinear combination (ql-combination) of $\left\{x_{k}\right\}_{k=1}^{n}$ corresponding to scalars $\left\{\alpha_{k}\right\}_{k=1}^{n} \subset \mathbb{R}$. ql-combination of $\left\{x_{k}\right\}_{k=1}^{n}$ corresponding to the scalars $\left\{\alpha_{k}\right\}_{k=1}^{n}$ may not be unique, from the definition, while it is well known that the 
linear combination of $\left\{x_{k}\right\}_{k=1}^{n}$ corresponding to these scalars is unique. The set

$$
\begin{aligned}
& \operatorname{Qsp} A=\left\{x \in X: \sum_{k=1}^{n} \alpha_{k} x_{k} \leq x, x_{1}, x_{2}, \ldots, x_{n}\right. \\
& \left.\quad \in A, \alpha_{1}, \alpha_{2}, \ldots, \alpha_{n} \in \mathbb{R}\right\}
\end{aligned}
$$

is said to be quasispan of $A$ and is denoted by Qsp $A$. One can see easily that Qsp $A$ is a subspace of $X$ [7].

It is clear that $\operatorname{Sp} A \subseteq \mathrm{Qsp} A$ and $\operatorname{Sp} A=\mathrm{Qsp} A$ iff $X$ is a linear space, where $\mathrm{Sp} A$ is the span of $A$ in $X$.

Definition 21. Let $X$ be a QLS, $\left\{x_{k}\right\}_{k=1}^{n} \subset X$ and $\left\{\lambda_{k}\right\}_{k=1}^{n} \subset \mathbb{R}$. If

$$
\theta \leq \lambda_{1} x_{1}+\lambda_{2} x_{2}+\cdots+\lambda_{n} x_{n}
$$

implies $\lambda_{1}=\lambda_{2}=\cdots=\lambda_{n}=0$, then $\left\{x_{k}\right\}_{k=1}^{n}$ is said to be quasilinear independent (ql-independent); otherwise, it is said to be ql-dependent in $X[7]$.

Theorem 22. Any set which has $n+1$ elements has to be qldependent in $\Omega_{C}\left(\mathbb{R}^{n}\right)$ [7].

Definition 23. Let $X$ be a QLS and let $A$ be a ql-independent subset of $X$. If $\mathrm{Qsp} A=X$, then the set $A$ is called a (Hamel) basis for $X[7]$.

Definition 24. Singular dimension of QLS $X$ is defined as the maximum number of ql-independent elements in singular subspace of $X$. If this number is finite then $X$ is called finite singular dimensional; otherwise it is called infinite singular dimensional. Further the dimension of regular subspace of $X$ is called regular dimension of $X$. It is denoted by $s-\operatorname{dim} X$ and $r-\operatorname{dim} X$, respectively [6].

On the other hand, if $s-\operatorname{dim} X=r-\operatorname{dim} X=a$ then $a$ is said to be dimension of $X$ and is written as $\operatorname{dim} X=a$ [6].

Definition 25. Let $X$ be a solid-floored quasilinear space. A Schauder basis is a sequence $\left(x_{n}\right)$ of elements of $X$ such that for every element $y \in X$ and for every $z \in F_{y}$ there exists a unique sequence of $\left\{\alpha_{n}\right\}$ scalars so that

$$
\begin{aligned}
& z=\sum_{n=1}^{\infty} \alpha_{n}^{(z)} x_{n}, \\
& y=\sup \left\{x \in X_{r}: x \leq y\right\} .
\end{aligned}
$$

This definition is identical to the following definition. $\left(x_{n}\right)$ is a Schauder basis of $X$ if and only if

$$
\begin{aligned}
h\left(z, \sum_{n=1}^{k} \alpha_{n}^{(z)} x_{n}\right) & \longrightarrow 0 \quad(k \longrightarrow \infty), \\
y & =\sup \left\{x \in X_{r}: x \leq y\right\}
\end{aligned}
$$

for every $y \in X$ and for every $z \in F_{y}$.
Example 26. $\Omega(\mathbb{R})$ and $\Omega_{C}(\mathbb{R})$ are solid-floored quasilinear space. But singular subspace of $\Omega_{C}(\mathbb{R})$ is a non-solid-floored quasilinear space.

Recall that the closed interval denoted by $[a, b]$ is the set of real numbers given by $[a, b]=\{x \in \mathbb{R}: a \leq x \leq$ $b$ \}. Although various other types of intervals (open, halfopen) appear throughout mathematics, our work will center primarily on closed intervals. In this paper, the term interval will mean closed interval.

\section{Interval Spaces}

In this section, we introduce some new examples of quasilinear space. Let $I \mathbb{R}$ be a set of all closed intervals of real numbers. In this case, $I \mathbb{R}$ is the whole compact convex subset of real numbers. Now, we can give two-dimensional interval vector such that $I \mathbb{R}^{2}=\left\{\left(X_{1}, X_{2}\right): X_{1}, X_{2} \in I \mathbb{R}\right\}$. And, so we can give

$$
I \mathbb{R}^{n}=\left\{\left(X_{1}, X_{2}, \ldots, X_{n}\right): X_{1}, X_{2}, \ldots, X_{n} \in I \mathbb{R}\right\} .
$$

We should state that $I \mathbb{R}^{n}$ is not a vector space that is not linear space. Also, the convergence of these sets was discussed in [8].

Example 27. Let $X=\left(X_{1}, X_{2}, \ldots, X_{n}\right) \in I \mathbb{R}^{n}$ and $Y=\left(Y_{1}, Y_{2}\right.$, $\left.\ldots, Y_{n}\right) \in I \mathbb{R}^{n}$. The algebraic sum operation on $I \mathbb{R}^{n}$ is defined by the expression

$$
X+Y=\left(X_{1}+Y_{1}, X_{2}+Y_{2}, \ldots, X_{n}+Y_{n}\right)
$$

and multiplication by a real number $\alpha \in \mathbb{R}$ is defined by

$$
\alpha \cdot X=\left(\alpha \cdot X_{1}, \alpha \cdot X_{2}, \ldots, \alpha \cdot X_{n}\right) .
$$

If we will assume that the partial order on $I \mathbb{R}^{n}$ is given by

$$
X \leq Y \Longleftrightarrow X_{i} \leq Y_{i} \quad 1 \leq i \leq n
$$

then $I \mathbb{R}^{n}$ is a quasilinear space with the above sum operation, multiplication, and partial order relation. From here, we get that $I \mathbb{R}^{n}$ is another example of quasilinear spaces.

Remark 28. The quasilinear space $I \mathbb{R}^{n}$ and the quasilinear space $\Omega_{C}\left(\mathbb{R}^{n}\right)$ are different from each other. For example, while the set $A=\left\{(x, y): x^{2}+y^{2} \leq 1\right\}$ is an element of $\Omega_{C}\left(\mathbb{R}^{2}\right)$, it is not an element of $I \mathbb{R}^{2}$. On the other hand, $B=([1,2],\{2\}) \in I \mathbb{R}^{2}$ but $B \notin \Omega_{C}\left(\mathbb{R}^{2}\right)$. So, $I \mathbb{R}^{n}$ and $\Omega_{C}\left(\mathbb{R}^{n}\right)$ are two different examples of quasilinear spaces.

Example 29. Let $A=(\{1\},\{0\}) \subset I \mathbb{R}^{2}$. We can find $-A=$ $(\{-1\},\{0\})$ such that $A+(-A)=(\{0\},\{0\})$. So, $A$ is a regular subset of $I \mathbb{R}^{2}$. Also, if $B_{i} \in \mathbb{R}$ for every $B=\left(B_{1}, B_{2}, \ldots, B_{n}\right) \in$ $I \mathbb{R}^{n}$, then $B$ is a regular subset of $I \mathbb{R}^{n}$. On the other hand, if $a_{i} \neq b_{i}$ for $1 \leq i \leq n$ and $a_{i}, b_{i} \in \mathbb{R}$, then $B=\left(\left[a_{1}, b_{1}\right]\right.$, $\left.\left[a_{2}, b_{2}\right], \ldots,\left[a_{n}, b_{n}\right]\right)$ is a singular subset of $I \mathbb{R}^{n}$.

The norm on $I \mathbb{R}^{n}$ is defined by

$$
\|X\|=\left\|\left(X_{1}, X_{2}, \ldots, X_{n}\right)\right\|=\sup _{1 \leq i \leq n}\left\|X_{i}\right\|_{I \mathbb{R}} .
$$


Then $I \mathbb{R}^{n}$ is a normed quasilinear space. From [1], we know that $I \mathbb{R}$ is a normed quasilinear space with $\|X\|=\sup _{x \in X}|x|$. For example, let $A=([1,3],[2,3],[-1,0]) \in I \mathbb{R}^{3}$. Then, we get $\|A\|=\sup \left\{\|[1,3]\|_{I \mathbb{R}},\|[2,3]\|_{I \mathbb{R}},\|[-1,0]\|_{I \mathbb{R}}\right\}=3$.

The Hausdorff metric is defined as usual:

$$
\begin{aligned}
& h_{I \mathbb{R}}(A, B) \\
& \quad=\inf \left\{r \geq 0: A \subseteq B+S_{r}(\theta), B \subseteq A+S_{r}(\theta)\right\},
\end{aligned}
$$

where $S_{r}(\theta)$ is the closed ball of radius $r$ about $\theta \in X$.

Theorem 30. $I \mathbb{R}^{n}$ interval space is a Banach $\Omega$-space with $\|X\|=\sup _{1 \leq i \leq n}\left\|X_{i}\right\|_{I \mathbb{R}}$.

Proof. Let $X^{n}$ be any Cauchy sequence in $I \mathbb{R}^{n}$. Then, given any $\epsilon>0$, there is $N$ such that for all $m, n>N$ we have

$$
\begin{gathered}
X^{n} \leq X^{m}+A^{1 \epsilon}, \\
X^{m} \leq X^{n}+A^{2 \epsilon}, \\
\left\|A^{j \epsilon}\right\| \leq \epsilon, \quad j=1,2 .
\end{gathered}
$$

Hence, for any $1 \leq i \leq n$, we get $\left\|A_{i}^{j \epsilon}\right\| \leq \epsilon$. On the other hand, since $I \mathbb{R}^{n}$ is a quasilinear space,

$$
\begin{aligned}
& X_{i}^{n} \leq X_{i}^{m}+A_{i}^{1 \epsilon}, \\
& X_{i}^{m} \leq X_{i}^{n}+A_{i}^{2 \epsilon}
\end{aligned}
$$

for every $1 \leq i \leq n$. This shows that $X_{i}^{n}$ is a Cauchy sequence in $I \mathbb{R}$. By completeness of $I \mathbb{R}$, there is $X_{i} \in I \mathbb{R}$ such that $X_{i}^{n} \rightarrow X_{i}$ for all $i \in[1, n]$. Furthermore, since $X_{i} \in I \mathbb{R}$ for every $i \in[1, n], X=\left(X_{1}, X_{2}, \ldots, X_{n}\right) \in I \mathbb{R}^{n}$. From (28), by letting $m \rightarrow \infty$, we obtain

$$
\begin{gathered}
X_{i}^{n} \leq X_{i}+B_{i}^{1 \epsilon}, \\
X_{i} \leq X_{i}^{n}+B_{i}^{2 \epsilon}, \\
\left\|B_{i}^{j \epsilon}\right\|_{I \mathbb{R}} \leq \epsilon, \quad j=1,2,
\end{gathered}
$$

for all $n>n_{0}$. So, we get $\left\|B^{j \epsilon}\right\| \leq \epsilon$, since $\left\|B^{j \epsilon}\right\|=\sup \left\|B_{i}^{j \epsilon}\right\|_{I \mathbb{R}}$. Also, we obtain

$$
\begin{gathered}
X^{n} \leq X+B^{1 \epsilon}, \\
X \leq X^{n}+B^{2 \epsilon}
\end{gathered}
$$

for all $1 \leq i \leq n$. This means that the sequence $\left(X^{n}\right)$ converges to $X$ in $I \mathbb{R}^{n}$.

Now, we will show that $I \mathbb{R}^{n}$ is an $\Omega$-space. Suppose $B_{X}=$ $n$ units $([-1,1],[-1,1], \ldots,[-1,1])$. From the definition of $\Omega$-space, since

$$
\|X\|=\sup \left\{\left\|X_{1}\right\|,\left\|X_{2}\right\|, \ldots,\left\|X_{n}\right\|\right\} \leq\left\|B_{X}\right\|=1
$$

we have $\left\|X_{1}\right\| \leq 1,\left\|X_{2}\right\| \leq 1, \ldots,\left\|X_{n}\right\| \leq 1 \Leftrightarrow X_{1} \subseteq[-1,1]$, $X_{2} \subseteq[-1,1], \ldots, X_{n} \subseteq[-1,1]$. This proves the theorem.
Example 31. The space $I \mathbb{R}^{n}$ is a normed quasilinear space with norm defined by

$$
\|X\|_{2}=\left(\sum_{i=1}^{n}\left\|X_{i}\right\|_{I \mathbb{R}}^{2}\right)^{1 / 2} .
$$

The quasilinear space $I \mathbb{R}^{n}$ with the inner product

$$
\langle X, Y\rangle=\sum_{i=1}^{n}\left\langle X_{i}, Y_{i}\right\rangle_{I \mathbb{R}}
$$

is an inner product quasilinear space.

Theorem 32. $I \mathbb{R}^{n}$ is a Hilbert quasilinear space with $\|\cdot\|_{2}$ norm.

Proof. The norm in Example 31 is defined by $\|X\|_{2}=$ $\left(\sum_{i=1}^{n}\left\|X_{i}\right\|_{I \mathbb{R}}^{2}\right)^{1 / 2}$ and can be obtained from the inner product quasilinear in Example 31. Let $\left(X^{n}\right)$ be any Cauchy sequence in $I \mathbb{R}^{n}$. Then, given any $\epsilon>0$, there is $N$ such that for all $m, n>N$ we have

$$
\begin{gathered}
X^{n} \leq X^{m}+A^{1 \epsilon}, \\
X^{m} \leq X^{n}+A^{2 \epsilon}, \\
\left\|A^{j \epsilon}\right\| \leq \epsilon, \quad j=1,2 .
\end{gathered}
$$

Hence, for any $1 \leq i \leq n$, we get $\left\|A_{i}^{j \epsilon}\right\| \leq \epsilon$. On the other hand, since $I \mathbb{R}^{n}$ is a quasilinear space,

$$
\begin{aligned}
& X_{i}^{n} \leq X_{i}^{m}+A_{i}^{1 \epsilon}, \\
& X_{i}^{m} \leq X_{i}^{n}+A_{i}^{2 \epsilon}
\end{aligned}
$$

for every $1 \leq i \leq n$. This shows that $X_{i}^{n}$ is a Cauchy sequence in $I \mathbb{R}$. By completeness of $I \mathbb{R}$, there is $X_{i} \in I \mathbb{R}$ such that $X_{i}^{n} \rightarrow X_{i}$ for all $i \in[1, n]$. Furthermore, since $X_{i} \in I \mathbb{R}$ for every $i \in[1, n], X=\left(X_{1}, X_{2}, \ldots, X_{n}\right) \in I \mathbb{R}^{n}$. From the above inequality, by letting $m \rightarrow \infty$, we obtain

$$
\begin{aligned}
X_{i}^{n} & \leq X_{i}+B_{i}^{1 \epsilon}, \\
X_{i} & \leq X_{i}^{n}+B_{i}^{2 \epsilon}, \\
\left\|B_{i}^{j \epsilon}\right\|_{I \mathbb{R}} & \leq \epsilon^{l}=\frac{\epsilon}{\sqrt{n}}, \quad j=1,2,
\end{aligned}
$$

for all $n>n_{0}$. So, we get

$$
\begin{aligned}
X^{n} & \leq X+B^{1 \epsilon}, \\
X & \leq X^{n}+B^{2 \epsilon},
\end{aligned}
$$

and $\left\|B^{j \epsilon}\right\| \leq \epsilon$ for all $1 \leq i \leq n$. This means that the sequence $\left(X^{n}\right)$ is convergent to $X$ in $I \mathbb{R}^{n}$.

Example 33. Let $X=\left(I \mathbb{R}^{2}, \subseteq\right)$ and take the singleton $A=$ $\{([1,2],[-1,3])\}$ in $X$. The q-span of $A$ is

$$
\mathrm{Qsp} A=\left\{X \in I \mathbb{R}^{2}: \lambda \cdot([1,2],[-1,3]) \subseteq X, \lambda \in \mathbb{R}\right\} .
$$


For example, $([0,2],[-1,4]) \in \mathrm{Qsp} A$ since $([1,2],[-1,3]) \subseteq$ $([0,2],[-1,4])$ whereas $([-1,3],[1,2]) \notin$ Qsp $A$. Because there is no $\lambda \in \mathbb{R}$ such that $\lambda \cdot([1,2],[-1,3]) \subseteq([-1,3]$, $[1,2])$, clearly, Qsp $A \neq I \mathbb{R}^{2}$. Let $B=\{(\{1\},\{0\}),(\{0\},\{1\})\}$ be another element of $X$. For any $x \in X$, clearly, we can write $\lambda_{1} \cdot(\{1\},\{0\})+\lambda_{2} \cdot(\{0\},\{1\}) \subseteq x$ for some $\lambda_{1}, \lambda_{2} \in \mathbb{R}$. This means Qsp $B=X$.

Example 34. In $\left(I \mathbb{R}^{2}, \subseteq\right)$, let $A=\{([2,3],[-1,1])\}$ and $B=$ $\{([-1,1],[-1,0])\}$. Then $A$ is ql-independent since $(\{0\},\{0\}) \subseteq$ $\lambda \cdot([2,3],[-1,1])$ for $\lambda=0$, where $(\{0\},\{0\})$ is the zero of the quasilinear space $I \mathbb{R}^{2}$. In contrast, the singleton $B$ is qldependent since $(\{0\},\{0\}) \subseteq \beta \cdot([-1,1],[-1,0])$ for $\beta=1$. This is an unusual case in linear spaces because a nonzero singleton is clearly a linearly independent set in such case. Particularly, any subset of $I \mathbb{R}^{2}$ possessing every vector of an element related to zero must be ql-dependent even if it is a singleton.

Example 35. From Example 33, Qsp $B=I \mathbb{R}^{2}$. Also, $B$ is qlindependent since $(\{0\},\{0\}) \subseteq \alpha_{1} \cdot(\{1\},\{0\})+\alpha_{2} \cdot(\{0\},\{1\})$. From here, $B=\{(\{1\},\{0\}),(\{0\},\{1\})\}$ is a basis for $I \mathbb{R}^{2}$.

Corollary 36. $I \mathbb{R}^{2}$ is a normed quasilinear space with $\|X\|_{2}=$ $\left(\sum_{i=1}^{2}\left\|X_{i}\right\|_{I \mathbb{R}}^{2}\right)^{1 / 2}$. From Example $31, I \mathbb{R}^{2}$ is an inner product quasilinear space with (33). Further, the set $\{(\{1\},\{0\}),(\{0\}$, $\{1\})\}$ is an orthonormal subset of $I \mathbb{R}^{2}$. Hence, $\{(\{1\},\{0\}),(\{0\}$, $\{1\})\}$ is an orthonormal basis for $I \mathbb{R}^{2}$.

Example 37. Let $A=([-1,1],[0,2]) \in I \mathbb{R}^{2}$. Floor of $A$ is

$$
\begin{aligned}
F_{A} & =\left\{\left(y_{1}, y_{2}\right) \in\left(I \mathbb{R}^{2}\right)_{r}:\left(y_{1}, y_{2}\right) \subseteq A\right\} \\
& =\left\{\left(y_{1}, y_{2}\right) \in \mathbb{R}^{2}:-1 \leq y_{1} \leq 1,0 \leq y_{2} \leq 2\right\} .
\end{aligned}
$$

Floor of $X=\left(X_{1}, X_{2}, \ldots, X_{n}\right) \in I \mathbb{R}^{n}$ is

$$
\begin{aligned}
& F_{I \mathbb{R}^{n}}=\bigcup F_{X}=\bigcup\left\{\left(y_{1}, y_{2}, \ldots, y_{n}\right) \in\left(I \mathbb{R}^{n}\right)_{r}: y_{1}\right. \\
& \left.\quad \in X_{1}, y_{2} \in X_{2}, \ldots, y_{n} \in X_{n}\right\} .
\end{aligned}
$$

Theorem 38. The space $I \mathbb{R}^{n}$ is a solid-floored quasilinear space.

Proof. Let $X \in I \mathbb{R}^{n}$. We know that $X=\left(X_{1}, X_{2}, \ldots, X_{n}\right)$ and $X_{i} \in I \mathbb{R}$ for all $1 \leq i \leq n$. From the definition of the floor of an element, we have

$$
F_{X}=\left\{Y \in\left(I \mathbb{R}^{n}\right)_{r}: Y \leq X\right\}
$$

Since $I \mathbb{R}^{n}$ is a quasilinear space, if $Y \leq X$, then we find $Y_{i} \leq X_{i}$ for every $1 \leq i \leq n$. Here, $X_{i} \in I \mathbb{R}$ for every $X=$ $\left(X_{1}, X_{2}, \ldots, X_{n}\right) \in I \mathbb{R}^{n}$ and $1 \leq i \leq n$. Since $Y \in \mathbb{R}^{n}, Y_{i} \in \mathbb{R}$ for all $i \in[1, n]$. Hence,

$$
X_{i}=\sup F_{X_{i}}=\sup \left\{Y_{i} \in \mathbb{R}: Y_{i} \leq X_{i}\right\}
$$

since $I \mathbb{R}$ is a solid-floored quasilinear space. This shows that $X=\sup F_{X}$ for every $X=\left(X_{1}, X_{2}, \ldots, X_{n}\right) \in I \mathbb{R}^{n}$.
Example 39. The set $W=\left\{X=\left(X_{1}, X_{2}, \ldots, X_{n}\right): X_{1}, X_{2}\right.$, $\left.\ldots, X_{n} \in(I \mathbb{R})_{d}\right\}$ consists of all symmetric elements of $I \mathbb{R}^{n}$. $W$ is a non-solid-floored quasilinear space since supremum of floors of every element in $W$ is $\{0\}$. For the same reason, the subspace of $I \mathbb{R}^{n}$

$$
\begin{aligned}
& V \\
& =\left\{X=\left(X_{1}, X_{2}, \ldots, X_{n}\right): X_{1}, X_{2}, \ldots, X_{n} \in(I \mathbb{R})_{o d}\right\}
\end{aligned}
$$

is non-solid-floored.

Lemma 40. The space $I \mathbb{R}^{n}$ is a proper quasilinear space.

Proof. Let us take arbitrary elements $A=$ $\left(A_{1}, A_{2}, \ldots, A_{n}\right), B=\left(B_{1}, B_{2}, \ldots, B_{n}\right) \in I \mathbb{R}^{n}$ such that $A \neq B$. If $A \subsetneq B$, then $A_{i} \varsubsetneqq B_{i}$ for at least $i \in[1, n]$. Hence, there is at least $a_{i} \in A_{i}$ such that $a_{i} \notin B_{i}$. So $\left\{a_{i}\right\} \subseteq A_{i}$ and $\left\{a_{i}\right\} \subsetneq B_{i}$. From here, we have

$$
\begin{gathered}
\left(\left\{a_{1}\right\},\left\{a_{2}\right\}, \ldots,\left\{a_{n}\right\}\right) \subseteq\left(A_{1}, A_{2}, \ldots, A_{n}\right), \\
\left(\left\{a_{1}\right\},\left\{a_{2}\right\}, \ldots,\left\{a_{n}\right\}\right) \subsetneq\left(B_{1}, B_{2}, \ldots, B_{n}\right) .
\end{gathered}
$$

If $B \varsubsetneqq A$, then $B_{i} \varsubsetneqq A_{i}$ for at least $i \in[1, n]$. Hence, there is at least $b_{i} \in B_{i}$ such that $b_{i} \notin A_{i}$. So $\left\{b_{i}\right\} \subseteq B_{i}$ and $\left\{b_{i}\right\} \subsetneq A_{i}$. From here, we have $\left(\left\{b_{1}\right\},\left\{b_{2}\right\}, \ldots,\left\{b_{n}\right\}\right) \subseteq\left(B_{1}, B_{2}, \ldots, B_{n}\right)=B$ and $\left(\left\{b_{1}\right\},\left\{b_{2}\right\}, \ldots,\left\{b_{n}\right\}\right) \subsetneq\left(A_{1}, A_{2}, \ldots, A_{n}\right)=A$. If there is not a comparison between $A$ and $B$, then there is not a comparison between $A_{i}$ and $B_{i}$ for some $i \in[1, n]$. Hence, there exist two elements $a_{i} \in A_{i}, a_{i} \notin B_{i}$ and $b_{i} \in B_{i}$, $b_{i} \notin A_{i}$ for some $i \in[1, n]$. Thus $\left\{a_{i}\right\} \subseteq A_{i},\left\{a_{i}\right\} \subsetneq$ $B_{i}$ and $\left\{b_{i}\right\} \subseteq B_{i},\left\{b_{i}\right\} \subsetneq A_{i}$ for this $i \in[1, n]$. So, we have $\left(\left\{a_{1}\right\},\left\{a_{2}\right\}, \ldots,\left\{a_{n}\right\}\right) \subseteq A,\left(\left\{a_{1}\right\},\left\{a_{2}\right\}, \ldots,\left\{a_{n}\right\}\right) \subsetneq B$ and $\left(\left\{b_{1}\right\},\left\{b_{2}\right\}, \ldots,\left\{b_{n}\right\}\right) \subseteq B,\left(\left\{b_{1}\right\},\left\{b_{2}\right\}, \ldots,\left\{b_{n}\right\}\right) \subsetneq A$. So, $I \mathbb{R}^{n}$ is a proper quasilinear space.

Example 41. The singular subspace of $I \mathbb{R}^{n}$

$$
\begin{aligned}
& \left(I \mathbb{R}^{n}\right)_{s} \\
& =\{0\} \\
& \quad \cup\left\{\left(X_{1}, X_{2}, \ldots, X_{n}\right): X_{1}, X_{2}, \ldots, X_{n} \in(I \mathbb{R})_{s}\right\}
\end{aligned}
$$

is improper, because, in this space, floors of some elements may be empty set and floors of any two different elements may be the same.

Example 42. Regular and singular dimensions of the quasilinear spaces $\mathbb{R}^{n}, I \mathbb{R}^{n},\left(I \mathbb{R}^{n}\right)_{s}$, and $\left(I \mathbb{R}^{n}\right)_{r}$ are as follows:

$$
\begin{aligned}
r-\operatorname{dim}\left(\mathbb{R}^{n}\right) & =n, \\
s-\operatorname{dim}\left(\mathbb{R}^{n}\right) & =0, \\
r-\operatorname{dim}\left(I \mathbb{R}^{n}\right) & =n, \\
s-\operatorname{dim}\left(I \mathbb{R}^{n}\right) & =n, \\
r-\operatorname{dim}\left(I \mathbb{R}^{n}\right)_{s} & =0, \\
s-\operatorname{dim}\left(I \mathbb{R}^{n}\right)_{s} & =n,
\end{aligned}
$$




$$
\begin{aligned}
& r-\operatorname{dim}\left(I \mathbb{R}^{n}\right)_{r}=n, \\
& s-\operatorname{dim}\left(I \mathbb{R}^{n}\right)_{r}=0 .
\end{aligned}
$$

Example 43. Let us consider the subspace $A=\left(I \mathbb{R}^{2}\right)_{s} \cup$ $\{(\{t\},\{0\}): t \in \mathbb{R}\}$ of $I \mathbb{R}^{2}$ and the elements $a_{1}=([1,2],\{0\})$ and $a_{2}=(\{0\},[2,4])$ of $A_{s}$. The set $\left\{a_{1}, a_{2}\right\}$ is ql-independent in $A_{s}$ since there are nonzero scalars $\lambda_{1}$ and $\lambda_{2}$ satisfying the inclusion $(\{0\},\{0\}) \subseteq \lambda_{1} a_{1}+\lambda_{2} a_{2}$. Hence singular dimension of $A$ must be greater than or equal to 2. Remember that $A$ is a subspace of $I \mathbb{R}^{2}$ and Theorem 22 . Then $s-\operatorname{dim} A=2$. Clearly, $A_{r}$ is equivalent to $\mathbb{R}$ and so $r-\operatorname{dim} A=1$.

\section{Interval Sequence Spaces}

In this section, we will introduce interval sequence spaces as a different example of quasilinear spaces. We first define the following new interval spaces. The set

$$
\begin{aligned}
I s & =\left\{X=\left(X_{n}\right) \in I \mathbb{R}^{\infty}:\left(X_{n}\right)=\left(X_{1}, X_{2}, \ldots, X_{n}, \ldots\right)\right. \\
& \left.\in I \mathbb{R}^{\infty}\right\}
\end{aligned}
$$

is called all intervals sequence space. Let $X=\left(X_{1}, X_{2}, \ldots\right.$, $\left.X_{n}\right) \in I \mathbb{R}^{n}$ and $Y=\left(Y_{1}, Y_{2}, \ldots, Y_{n}\right) \in I \mathbb{R}^{n}$. The algebraic sum operation on $I s$ is defined by the expression

$$
X+Y=\left(X_{1}+Y_{1}, X_{2}+Y_{2}, \ldots, X_{n}+Y_{n}, \ldots\right)
$$

and multiplication by a real number $\alpha \in \mathbb{R}$ is defined by

$$
\alpha \cdot X=\left(\alpha \cdot X_{1}, \alpha \cdot X_{2}, \ldots, \alpha \cdot X_{n}, \ldots\right) .
$$

If we will assume that the partial order on $I s$ is given by

$$
X \leq Y \Longleftrightarrow X_{i} \leq Y_{i} \quad 1 \leq i \leq n
$$

then $I s$ is a quasilinear space with the above sum operation, multiplication, and partial order relation. Is is a metric space with

$$
d(X, Y)=\sum_{i=1}^{\infty} \frac{1}{2^{i}}\left[\frac{h\left(X_{i}, Y_{i}\right)}{1+h\left(X_{i}, Y_{i}\right)}\right]
$$

for every $X, Y \in I s$. Here, $h$ is the known Hausdorff metric on $I \mathbb{R}$.

Now, we give a new interval sequence space, denoted by $I l_{\infty}$

$$
I l_{\infty}=\left\{X=\left(X_{n}\right) \in I \mathbb{R}^{\infty}:\left(X_{n}\right) \text { bounded }\right\},
$$

namely, the space of all bounded interval sequences $\left(X_{n}\right)$ of real numbers such that

$$
\sup \left\{\left\|X_{i}\right\|_{I \mathbb{R}}\right\} \leq \infty \Longleftrightarrow X \in I l_{\infty} .
$$

Here, the boundedness of interval sequences will be considered according to the Hausdorff metric. $I l_{\infty}$ is a quasilinear space with (48), (49), and (50). The sum operation and scalar multiplication are well defined since

$$
\begin{aligned}
\sup \left\{\left\|X_{i}+Y_{i}\right\|_{I \mathbb{R}}\right\} & \leq \sup \left\{\left\|X_{i}\right\|_{I \mathbb{R}}+\left\|Y_{i}\right\|_{I \mathbb{R}}\right\} \\
& \leq \sup \left\{\left\|X_{i}\right\|_{I \mathbb{R}}\right\}+\sup \left\{\left\|Y_{i}\right\|_{I \mathbb{R}}\right\} \\
& \leq \infty, \\
\sup \left\{\left\|\lambda X_{i}\right\|_{I \mathbb{R}}\right\} & \leq \sup \left\{\lambda\left\|X_{i}\right\|_{I \mathbb{R}}\right\} \leq \lambda \sup \left\{\left\|X_{i}\right\|_{I \mathbb{R}}\right\} \\
& \leq \infty .
\end{aligned}
$$

$I l_{\infty}$ is a normed quasilinear space with norm function defined by

$$
\|X\|=\sup \left\{\left\|X_{n}\right\|_{I \mathbb{R}}: n \in \mathbb{N}\right\} .
$$

Indeed, since it is easy to verify conditions (14)-(17) we only prove condition (18). Let $X \leq Y+X_{\epsilon}$ and $\left\|X_{\epsilon}\right\| \leq \epsilon$ for every $X, Y \in I l_{\infty}$. Then we have $\left\|X_{\epsilon_{i}}\right\|_{I \mathbb{R}} \leq \epsilon$ for all $1 \leq i \leq \infty$. On the other hand, we find $X_{i} \leq Y_{i}+X_{\epsilon_{i}}$ for all $1 \leq i \leq \infty$ when $X \leq Y+X_{\epsilon}$. Since $I \mathbb{R}$ is a normed quasilinear space, we get $X_{i} \leq Y_{i}$ for every $i \in[1, \infty]$. Thus, we obtain $X \leq Y$.

Denote

$$
I c_{0}=\left\{X=\left(X_{n}\right) \in I \mathbb{R}^{\infty}:\left(X_{n}\right) \longrightarrow 0\right\} ;
$$

that is, the space $I c_{0}$ consists of all convergent sequences $\left(X_{n}\right)$ whose limit is zero such that

$$
\lim _{n \rightarrow \infty}\left\|X_{n}\right\|_{I \mathbb{R}}=0 \Longleftrightarrow X \in I c_{0} .
$$

$I c_{0}$ is a quasilinear space the same as $I l_{\infty}$ with (48), (49), and (50).

The space $I c_{0}$ with the norm

$$
\|X\|=\sup _{i \in \mathbb{N}}\left\|X_{i}\right\|_{I \mathbb{R}}
$$

is a normed quasilinear space.

A new example of the nonlinear quasilinear space is defined by

$$
I l_{2}=\left\{X=\left(X_{n}\right) \in I \mathbb{R}^{\infty}: \sum_{i=1}^{\infty}\left\|X_{n}\right\|_{I \mathbb{R}}^{2} \text { bounded }\right\} .
$$

$I l_{2}$ is a not linear space.

Example 44. $\mathrm{Il}_{2}$ is a metric space with

$$
\begin{aligned}
& d(X, Y) \\
& \quad=\inf \left\{r \geq 0: X \subseteq Y+S_{r}(\theta), Y \subseteq X+S_{r}(\theta)\right\},
\end{aligned}
$$

where $S_{r}(\theta)=\left\{X \in I l_{2}:\|X\| \leq r\right\}$.

Proposition 45. $\mathrm{Il}_{2}$ with the norm defined by

$$
\|X\|=\left(\sum_{i=1}^{\infty}\left\|X_{i}\right\|_{I \mathbb{R}}^{2}\right)^{1 / 2}
$$

is a normed quasilinear space but $\mathrm{Il}_{2}$ is not a Banach quasilinear space with (61). 
Proof. First we show that $I l_{2}$ is a quasilinear space, then that (61) is a norm in $I_{2}$, and finally that the space is not complete. The fact that $I_{2}$ is a quasilinear space with (48), (49), and (50) is obvious. Here, sum operation and scaler multiplication are well defined since

$$
\begin{aligned}
& \left(\sum_{n=1}^{\infty}\left\|X_{n}+Y_{n}\right\|_{I \mathbb{R}}^{2}\right)^{1 / 2} \\
& \leq\left(\sum_{n=1}^{\infty}\left(\left\|X_{n}\right\|_{I \mathbb{R}}+\left\|Y_{n}\right\|_{I \mathbb{R}}\right)^{2}\right)^{1 / 2} \\
& \leq\left(\sum_{n=1}^{\infty}\left\|X_{n}\right\|_{I \mathbb{R}}^{2}\right)^{1 / 2}+\left(\sum_{n=1}^{\infty}\left\|Y_{n}\right\|_{I \mathbb{R}}^{2}\right)^{1 / 2}<\infty, \\
& \|\lambda X\|=\left(\sum_{n=1}^{\infty}\left\|\lambda X_{n}\right\|_{I \mathbb{R}}^{2}\right)^{1 / 2}=\lambda\left(\sum_{n=1}^{\infty}\left\|X_{n}\right\|_{I \mathbb{R}}^{2}\right)^{1 / 2}<\infty
\end{aligned}
$$

for every $X, Y \in I_{2}$ and $\lambda \in \mathbb{R}$. Also, (61) provides the (14)(18) conditions and $\mathrm{Il}_{2}$ is a normed quasilinear space with (61).

Now, we assume that $X^{n}$ is a Cauchy sequence in $I l_{2}$. If

$$
X^{n}=\left(X_{1}^{n}, X_{2}^{n}, X_{3}^{n}, \ldots\right)
$$

then given any $\epsilon>0$, there exists a number $n_{0}$ such that

$$
d\left(X_{i}^{n}, X_{i}^{m}\right) \leq \epsilon
$$

Namely, for every $\epsilon>0$, there exists a number $n_{0}$ such that

$$
\begin{gathered}
X_{i}^{n} \leq X_{i}^{m}+A_{i}^{1 \epsilon}, \\
X_{i}^{m} \leq X_{i}^{n}+A_{i}^{2 \epsilon} .
\end{gathered}
$$

Note that this implies that for every fixed $i \in \mathbb{N}$ and for every $\epsilon>0$ there exists a number $n_{0}$ such that

$$
\left\|A_{i}^{j \epsilon}\right\|_{I \mathbb{R}} \leq \epsilon .
$$

But this means that, for every $i$, the sequence $\left(X_{i}^{n}\right)$ is a Cauchy sequence in $I \mathbb{R}$ and thus convergent. Denote

$$
\begin{gathered}
X_{i}^{n} \longrightarrow X_{i} \quad i=1,2, \ldots, \\
X=\left(X_{1}, X_{2}, \ldots\right) .
\end{gathered}
$$

We are not going to prove that $X$ is an element of $I l_{2}$ and that the sequence $\left(X^{n}\right)$ does not converge to $X$. Indeed, from (65), by letting $m \rightarrow \infty$, we obtain

$$
\begin{aligned}
X_{i}^{n} & \leq X_{i}+B_{i}^{1 \epsilon}, \\
X_{i} & \leq X_{i}^{n}+B_{i}^{2 \epsilon}, \\
\left\|B_{i}^{j \epsilon}\right\|_{I \mathbb{R}} & \leq \epsilon^{\imath}
\end{aligned}
$$

for every $n \geq n_{0}$. Here, for every $i$, we get

$$
\begin{gathered}
X^{n} \leq X+B^{1 \epsilon}, \\
X \leq X^{n}+B^{2 \epsilon}
\end{gathered}
$$

but

$$
\left\|B^{j \epsilon}\right\| \not \epsilon .
$$

So, we have that $X^{n}$ is not convergent to $X$ whether or not $\sum_{i=1}^{\infty}\left\|X_{i}\right\|^{2} \leq \infty$.

Corollary 46. $\mathrm{Il}_{2}$ interval sequence space is not a Banach quasilinear space with (61) since

$$
X^{n} \longrightarrow X \Longrightarrow X_{i}^{n} \longrightarrow X_{i}
$$

but

$$
X_{i}^{n} \longrightarrow X_{i} \nrightarrow X^{n} \longrightarrow X
$$

for every $X^{n}=\left(X_{1}^{n}, X_{2}^{n}, \ldots\right)$.

Example 47. $I l_{2}, I c_{0}$, and $I l_{\infty}$ interval sequence spaces are solid-floored quasilinear space. Definition of the $I_{2}$ interval sequence space $X=\left(X_{n}\right) \in I l_{2} \Leftrightarrow \sum_{n=1}^{\infty}\left\|X_{n}\right\|_{I \mathbb{R}}^{2}<\infty$. We find definition of solid-flooredness in which

$$
F_{X}=\left\{Y \in\left(I l_{2}\right)_{r}: Y \leq X\right\} .
$$

Since $I l_{2}$ is a quasilinear space, we find $Y_{i} \in \mathbb{R}$ and $X_{i} \in I \mathbb{R}$ such that

$$
Y_{i} \leq X_{i}
$$

for every $1 \leq i<\infty$. Here, we see clearly that $Y_{i} \in F_{X_{i}}$. Otherwise, since $I \mathbb{R}$ is a solid-floored quasilinear space, we have

$$
X_{i}=\sup F_{X_{i}}=\left\{Y_{i} \in \mathbb{R}: Y_{i} \leq X_{i}\right\}
$$

for every $i$. Hence, we obtain $X=\sup F_{X}$ since $I l_{2}$ is a quasilinear space with " $\leq$ " relation.

Example 48. Singular subspace of $I c_{0}$ is a non-solid-floored quasilinear space. For example, we have $\sup F_{B}=(0,0, \ldots)=$ 0 for $B=([-1,1], 0,0, \ldots) \in\left(I c_{0}\right)_{s}$.

Example 49. Let us recall that $I c_{0}$ is a quasilinear space with the partial order relation " $\subseteq$." If we take $X=\left(I c_{0}\right)_{s} \cup\{\emptyset\}$ where $\emptyset=(0,0, \ldots) \in c_{0}$, then $r-\operatorname{dim} X=0$ and $s-\operatorname{dim} X=\infty$. The quantity of ql-independent elements in $X$ is not finite. Indeed, the family

$$
\begin{aligned}
& \{([1,2], 0,0, \ldots),(0,[1,2], 0,0, \ldots), \ldots, \\
& \quad(0,0, \ldots,[1,2], 0, \ldots), \ldots\}
\end{aligned}
$$

is ql-independent. Let us show that any finite subset of this family is ql-independent. This also implies that this family is ql-independent: assume that

$$
\begin{aligned}
\emptyset= & \{(0,0, \ldots)\} \\
\subseteq & \lambda_{1}([1,2], 0,0, \ldots)+\lambda_{2}(0,[1,2], 0,0, \ldots)+\cdots \\
& +\lambda_{k}(0,0, \ldots,[1,2], 0, \ldots) \\
= & \left(\lambda_{1}[1,2], \lambda_{2}[1,2], \ldots, \lambda_{k}[1,2], 0,0, \ldots\right) \\
\Longleftrightarrow & \lambda_{1}=\lambda_{2}=\cdots=\lambda_{k}=0 .
\end{aligned}
$$


Example 50. For the QLS $X=I c_{0}, r-\operatorname{dim} X=\infty$ and $s-$ $\operatorname{dim} X=\infty$.

Example 51. We can say that $r-\operatorname{dim} X=2$ and $s-\operatorname{dim} X=\infty$, for the QLS

$X$

$$
\begin{aligned}
= & \left(I l_{\infty}\right)_{s} \\
& \cup\{(0,0, \ldots, 0, k, 0,0, \ldots, 0, l, 0,0, \ldots): k, l \in \mathbb{R}\} .
\end{aligned}
$$

Theorem 52. Let $E_{1}=(\{1\},\{0\},\{0\},\{0\}, \ldots), E_{2}=(\{0\},\{1\}$, $\{0\},\{0\}, \ldots), \ldots$ The sequence $\left(E_{n}\right)$ is a Schauder basis for I $c_{0}$ and $I l_{p}$ for $(p \leq 1)$.

Proof. Now, we show that $\left(E_{n}\right)$ is a Schauder basis for $I c_{0}$; the other proof is similar to $I c_{0}$. Let $Y=\left(Y_{k}\right)_{k=1}^{\infty} \in I c_{0}$ and $Z=$ $\left(X_{1}, X_{2}, \ldots, X_{n}, \ldots\right) \in\left(I c_{0}\right)_{r}$ for every $Z \in F_{Y}$. We choose $\alpha_{1}=X_{1}, \alpha_{2}=X_{2}, \ldots, \alpha_{n}=X_{n}, \ldots$ From here, we get

$$
\begin{aligned}
\| Z & -\sum_{k=1}^{n} \alpha_{k} E_{k}\left\|_{I c_{0}}=\right\| Z-\sum_{k=1}^{n} X_{k} E_{k} \|_{I c_{0}} \\
& =\|\left(X_{1}, X_{2}, \ldots, X_{n}, \ldots\right) \\
& -\left(X_{1}, X_{2}, \ldots, X_{n},\{0\},\{0\}, \ldots\right) \| \\
& =\left\|\{0\},\{0\}, \ldots, X_{n+1}, X_{n+2}, \ldots\right\| \\
& =\sup _{k \geq n+1}\left|X_{k}\right| \longrightarrow 0 \quad(n \longrightarrow \infty) .
\end{aligned}
$$

Since

$$
h\left(Z, \sum_{k=1}^{n} \alpha_{k} E_{k}\right) \leq\left\|Z-\sum_{k=1}^{n} \alpha_{k} E_{k}\right\|_{I c_{0}}
$$

we have $h\left(Z, \sum_{k=1}^{n} \alpha_{k} E_{k}\right) \rightarrow 0$ for $(n \rightarrow \infty)$. Now we show that this representation is unique.

To prove uniqueness, we assume that

$$
\begin{aligned}
& Z=\sum_{k=1}^{\infty} \alpha_{k} E_{k}, \\
& Z=\sum_{k=1}^{\infty} \beta_{k} E_{k}
\end{aligned}
$$

for $\alpha_{k} \neq \beta_{k}, 1 \leq k<\infty$. Since $Z \in\left(I c_{0}\right)_{r}$, we get

$$
\begin{aligned}
0 & =\|Z-Z\|=\left\|\sum_{k=1}^{\infty} \alpha_{k} E_{k}-\sum_{k=1}^{\infty} \beta_{k} E_{k}\right\| \\
& =\left\|\left(\left\{\alpha_{1}-\beta_{1}\right\},\left\{\alpha_{2}-\beta_{2}\right\}, \ldots,\left\{\alpha_{n}-\beta_{n}\right\}, \ldots\right)\right\| \\
& =\sup _{1 \leq k<\infty}\left|\alpha_{k}-\beta_{k}\right| .
\end{aligned}
$$

Hence, we find $\alpha_{k}=\beta_{k}$ for every $1 \leq k<\infty$. This proves the theorem.

Example 53. $\left(E_{n}\right)$ sequence given in Theorem 52 is an orthonormal basis for $I c_{0}$.

\section{Competing Interests}

The authors declare that there are no competing interests regarding the publication of this paper.

\section{References}

[1] S. M. Aseev, "Quasilinear operators and their application in the theory of multivalued mappings," Trudy Matematicheskogo Instituta imeni VA Steklova, vol. 167, pp. 25-52, 1985.

[2] G. Alefeld and G. Mayer, "Interval analysis: theory and applications," Journal of Computational and Applied Mathematics, vol. 121, no. 1-2, pp. 421-464, 2000.

[3] V. Lakshmikantham, T. Gnana Bhaskar, and J. Vasundhara Devi, Theory of Set Differential Equations in Metric Spaces, Cambridge Scientific Publishers, Cambridge, UK, 2006.

[4] H. Bozkurt, S. Çakan, and Y. Yılmaz, "Quasilinear inner product spaces and Hilbert quasilinear spaces," International Journal of Analysis, vol. 2014, Article ID 258389, 2014.

[5] Y. Yılmaz, S. Çakan, and Ş. Aytekin, "Topological quasilinear spaces," Abstract and Applied Analysis, vol. 2012, Article ID 951374, 10 pages, 2012.

[6] S. Çakan and Y. Yılmaz, "Normed proper quasilinear spaces," Journal of Nonlinear Sciences and Applications, vol. 8, pp. 816836, 2015.

[7] H. K. Banaz1l, On quasilinear operators between quasilinear spaces [M.S. thesis], Malatya, Turkey, 2014.

[8] R. E. Moore, R. B. Kearfott, and M. J. Cloud, Introduction to Interval Analysis, Society for Industrial and Applied Mathematics, Philadelphia, Pa, USA, 2009. 


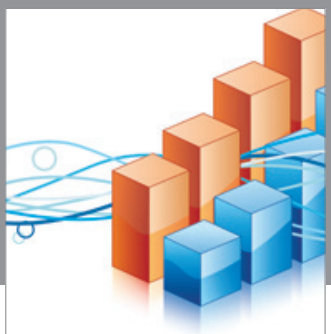

Advances in

Operations Research

vatem alat4

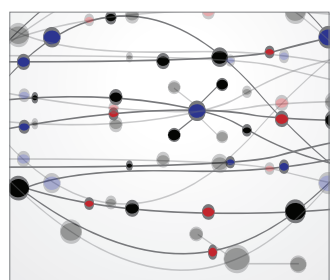

\section{The Scientific} World Journal
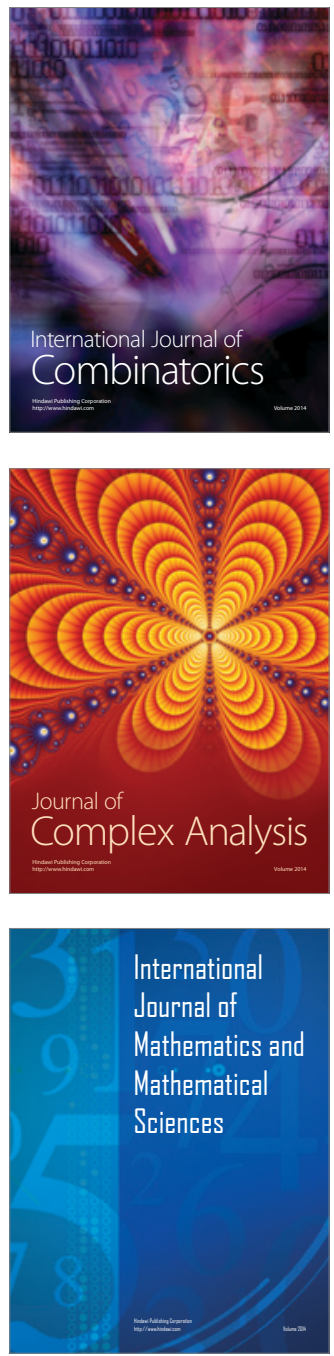
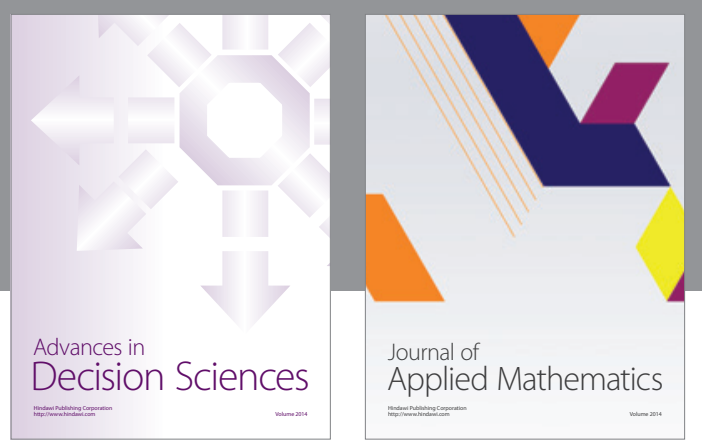

Algebra

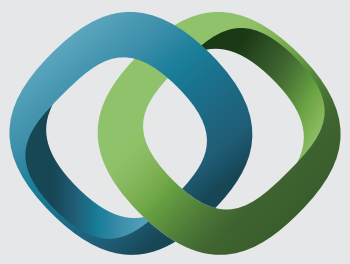

\section{Hindawi}

Submit your manuscripts at

http://www.hindawi.com
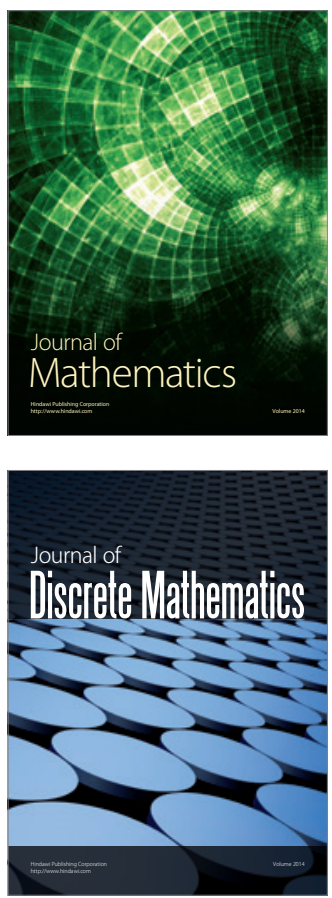

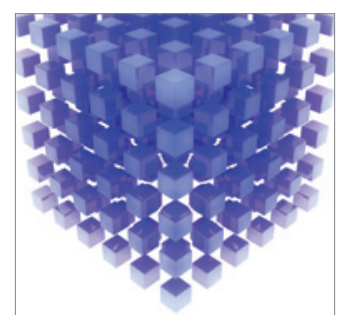

Mathematical Problems in Engineering
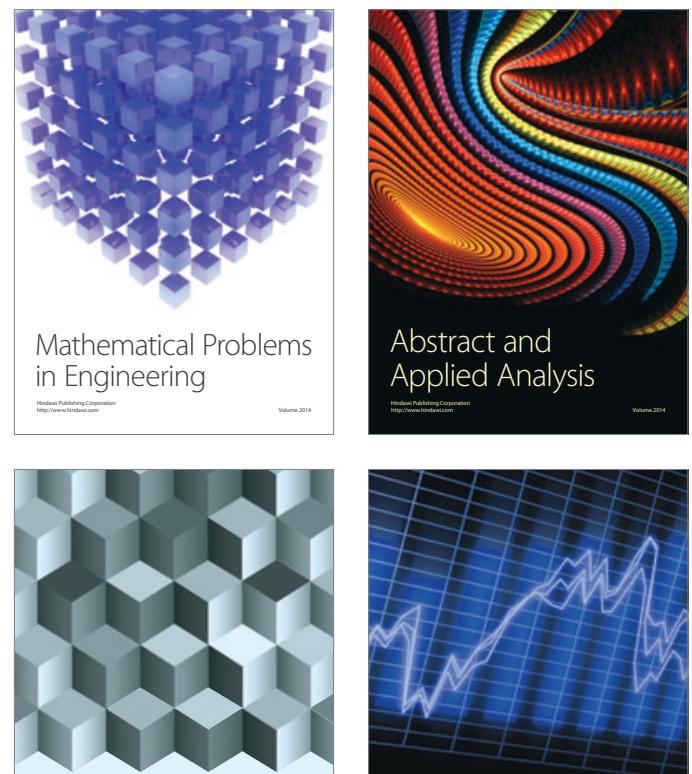

Journal of

Function Spaces

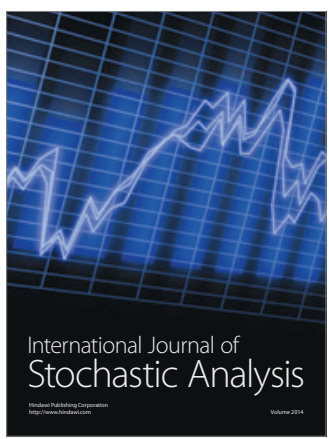

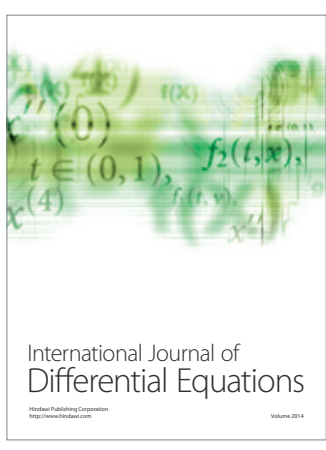
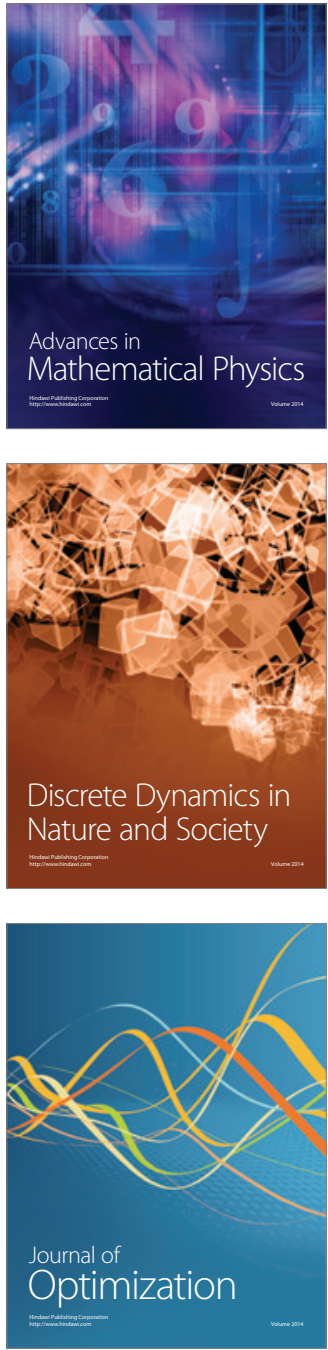